\title{
Pyogenic Granuloma of Gingiva: A Misnomer
}

\author{
Dr.Shrinidhi.M.S ${ }^{1}$, Dr. Usha Chikkaiah ${ }^{2}$ \\ Professor \& Head, Department of Periodontics, Sharavathi Dental College and Hospital, Shivamogga-577204, \\ Karnataka, India. \\ Reader, Department of Oral Medicine \& Radiology, Sharavathi Dental College and Hospital, Shivamogga- \\ 577204, Karnataka, India
}

\begin{abstract}
A varied spectrum of lesions ranging from developmental, reactive, inflammatory to neoplastic manifests in the oral cavity, as oral mucosa is constantly subjected to varied stimuli. These lesions may be either generalized or localized. Most of the localized oral overgrowths are considered to be reactive rather than neoplastic in nature. The reactive lesions of oral cavity are associated with injuries of soft tissue which stimulate an exuberant or excessive response and have high prevalence rates and different involvement pattern.

Key words: Reactive, Non-neoplastic, Oral mucosa, Localized overgrowth, Tissue injury.
\end{abstract}

\section{Introduction:}

Growth over the gingival tissues may be focal or generalized, often the result of an underlying systemic disease, drug-induced or local iatrogenic factors [1].The localized reactive hyperplastic lesions (LRHL) are often a diagnostic dilemma as they manifest clinically in a similar manner [1]. LRHL of gingiva are categorized into four sub-types like Focal Fibrous Hyperplasia (FFH), Pyogenic Granuloma (PG), Peripheral Ossifying Fibroma (POF) and Peripheral Giant Cell Granuloma (PGCG) [2]. The aim of this paper is to present one such case of localized reactive hyperplastic lesion of gingiva whose terminology is a misnomer.

\section{Case Report:}

A 55yr old female patient reported with a complaint of growth in her upper left side of mouth. She had initially noticed a small growth 9months back without any pain, but since 2 months the growth had increased in size, associated with pain and bleeding while brushing the teeth and chewing food. The mobile teeth in the same region was removed in a private clinic one month back to relieve the discomfort, however the lesion exacerbated and hence was referred to our OPD for further evaluation. Past medical history was non contributory. On clinical examination, no extraoral changes were seen. Intraoral examination revealed a solitary sessile growth measuring about $3.5 \mathrm{~cm} \mathrm{X} 4 \mathrm{~cm}$ on buccal gingiva with respect to the $25,26,27$ extending from the distal aspect of 24 to mesial aspect of 27 and extending downwards to the occlusal surface, leading to trauma on occlusion. The growth was smooth and erythematous (Figure 1), tender, semi-firm in consistency and showed bleeding spots on palpation. Clinically 26 was missing, and generalized mobility of teeth were present. A working diagnosis of pyogenic granuloma of gingiva was arrived at. The other differentials like peripheral giant cell granuloma, irritation fibroma, and peripheral ossifying fibroma were considered. Radiographic examination of the patient did not show any bony involvement suggestive of the soft tissue lesion. OPG showed generalized interdental bone loss suggestive of generalized periodontitis (Figure 2). Excision of the lesion was done under LA with removal of the adjacent mobile teeth and the specimen was sent for the histologic examination. The $\mathrm{H}$ \& E stained sections showed ulcerated epithelium with infilterated chronic inflammatory cells in the connective tissue stroma and numerous budding blood vessels suggestive of pyogenic granuloma (Figure 3). The patient was on regular follow-up, and the post excision healing was uneventful (Figure 4).

\section{Discussion:}

The term pyogenic granuloma is a misnomer as it is not associated with pus or a granulomatous lesion [3]. Hullihen in 1844, reported the first case in literature. In 1897, occurance of PG in man was first described as Botryomycosis hominis by Poneet and Dor [1,3]. The term Pyogenic granuloma or Granuloma pyogenicum was coined by Hartzell in 1904 [3]. Since then various terminologies were used like Crocker and Hartzells disease. Angelopoulous based on the histologic view, described PG as Hemangiomatous granuloma. Cawson and coworkers named it as Granuloma telangiectacticum. They described PG in 2 forms as lobular capillary hemangioma (LCH) and Non-LCH. The various other terminologies used were granuloma Pediculatum Benignum, Benign vascular tumor and during pregnancy as Granuloma gravidarum[1,3]. 


\subsection{Etiopathogenesis:}

Various etiological agents have been suggested to initiate PG, like chronic low grade irritation due to exfoliation of primary tooth, eruption of permanent teeth, defective or overhanging restoration, food impaction or tooth brush trauma, sharp tooth $[1,3,4]$. Other factors such as hormones, drugs, gingival inflammation and preexisting vascular lesions lead to the development of these lesions.

Various authors regarded PG as an infectious entity. Kerr and Bhaskar et al observed various Gram +ve and Gram-ve bacilli more commonly in ulcerated lesions near the surface tan in deeper aspects suggesting contamination from oral flora. Shafer et al stated that some minor trauma to tissue provided pathway for invasion of non-specific types of microorganisms[1,3]. The tissue responds to these low virulence organisms by overzealous proliferation of a vascular type of connective tissue. Reichart et al stated that granulation tissue formed due to irritation by contamination of oral flora become covered by fibrin mimicking pus. They also stated that still suppuration is not a characteristic in oral PG to support infectious origin. Regezi et al suggested oral PG representing an exuberant connective tissue proliferation to known stimulus or injury like calculus or foreign material within the gingival crevice[1,3]. In the present case, the main etiological cause could be the tooth accumulated materials and pre-existing periodontal disease which had led to the development of the lesion.

\subsection{Clinical features:}

Oral PG is the most common gingival overgrowth accounting for $75 \%$ occurrence on the gingiva, more commonly affecting the maxilla than the mandible. Again, the anterior than the posterior regions, and facial than the lingual surfaces being frequently involved. The other extra-gingival sites involved are lips, tongue and buccal mucosa[1,3,5]. It is predominantly seen in females in second decade of life with male to female predilection of $1: 1.5[3,5]$. In the present Case-study the occurrence of the lesion was in maxillary facial aspect of the gingiva in the posterior region.

PG is a smooth/lobulated exophytic lesion manifesting as small, red, erythematous papules either on a pedunculated or sessile base. The size of PG varies from few $\mathrm{mm}$ to several $\mathrm{cm}$ rarely exceeding $2.5 \mathrm{~cm}$ reaching its full size within weeks or months and remaining indefinitely thereafter[3,4]. Clinical development of the lesion is slow, sometimes rapid and asymptomatic. The color ranges from pink to red to purple depending on the age of the lesion. The surface is characteristically ulcerated and friable. The younger lesions are highly vascular causing considerable bleeding on minor trauma whereas the older lesions become more collagenized and pink $[3,4,5]$. Rarely PG causes any bony changes. Long standing lesions show localized erosion of alveolar bone[3,4].

\subsection{Differential diagnosis:}

Pyogenic granuloma of gingiva has to be differentiated from other reactive exophytic lesions like Peripheral Giant Cell Granuloma, Irritation Fibroma and Peripheral Ossifying Fibroma[2,6].

Peripheral giant cell granuloma (PGCG) involves gingiva or edentulous alveolar mucosa consisting of proliferating endothelial cells, rich capillary bed, chronic inflammatory cells, fibroblasts and multinucleated giant cells. Trauma is believed to be one of the etiologic factor. PGCG is more bluish purple compared to bright red pyogenic granuloma, most commonly seen in females with peak incidence in $5^{\text {th }}$ and $6^{\text {th }}$ decade. They may occur in both anterior and posterior region, in mandible. Radiographically cupping or resorption of underlying bone is noticed. Complete surgical removal is the treatment of choice with a recurrence of $10 \%[2,6]$.

The fibroma is most common reactive hyperplasia of fibrous connective tissue in response to local irritation or trauma. Most commonly seen on buccal mucosa, other sites being labial mucosa, tongue and gingiva. It is likely that many gingival fibromas represent fibrous maturation of preexisting pyogenic granuloma. They typically appears as a smooth surfaced pink nodule with a sessile base sometimes pedunculated. Surface may appear white due to hyperkeratosis. The size ranges from $1.5 \mathrm{~cm}$ or less in diameter, usually seen in $4^{\text {th }}$ to $6^{\text {th }}$ decade of life with male to female predilection of 1:2. Surgical excision is the treatment of choice with no recurrence rate[2,6].

Many considered pyogenic granuloma as an immature form of POF due to their similar clinical and histopathological features, whereas POF is firmer and less friable and typically shows a longer course which explains the calcification and or ossification secondary to fibroblasts maturation to collagen tissue. POF presents as pedunculated or sessile, red to pink mass with smooth or irregular surface. They are generally less than $2 \mathrm{~cm}$ in diameter. Most commonly seen in females, in maxillary anterior region. Radiographically POF varies from completely no changes to areas of calcification, superficial bone loss or cupping defect. Surgical excision is the treatment of choice with recurrence rate of $16 \%[2,6]$. 


\subsection{Histopathologic features:}

The histologic appearance of pyogenic granuloma is similar to that of granulation tissue except that it is exuberant and is usually well localized. Numerous small and larger endothelium lined channels are formed that are engorged with red blood cells. These vessels sometimes organized in lobular aggregates and Pathologists consider it as a lobular capillary hemangioma. The overlying epithelium if present is generally thin and atrophic, but may be hyperplastic. If the lesion is ulcerated it shows fibrinous exudates of varying thickness over the surface. The most striking feature is the presence of extreme proliferation of fibroblasts and budding endothelial cells with endothelium lined vascular spaces along with infilteration of inflammatory cells $[1,3]$.

\subsection{Treatment \& prognosis:}

Surgical excision is the treatment of choice and curettage of underlying tissue is recommended with removal of the local factors if present. Bhaskar and Jacoway reported the recurrence rate of $15.8 \%$ after conservative excision, probably due to the improper curettage or improper removal of the local etiological factors $[3,4,5]$.

\section{Conclusion:}

Although oral pyogenic granuloma is the most common non-neoplastic lesion of gingiva, early diagnosis and removal of these lesions along with elimination of the etiological factors is of utmost important to minimize potential dentoalveloar complications and to preserve and improve the health of mucogingival complex.

\section{CONFLICT OF INTEREST: NIL \\ 6. SOURCE OF FUNDING: NIL \\ 7. ACKNOWLEDGEMENT: NIL}

\section{References:}

[1]. Reet Kamal, Parveen Dahiya, Abhiney Puri. Oral pyogenic granuloma: various concepts of etiopathogenesis. J of Oral and Maxillofacial Pathology 16(1), 2012, 79-81.

[2]. Vandana Reddy, Susmita Saxena, Sanjeev Saxena, Munish Reddy. Reactive hyperplastic lesions of the oral cavity: A ten year observational study on North Indian population. J Clin Exp Dent 4(3), 2012, 36-40.

[3]. Hamid Jafarzadeh, Majid Sanatkhani and Nooshin Mohtasham. Oral pyogenic granuloma: A review. J of Oral Science 48(4), 2006, $167-175$.

[4]. Pushpendra Kumar Verma, Ruche Srivastava, H C Baranwal, T P Chhaturvedi, Anju Gautam And Amit Singh. Pyogenic granulomaHyperplastic lesion of the gingiva: Case Reports. The Open Dentistry Journal 6, 2012, 153-56.

[5]. Mythireyi D, K Vinayagavel. Management of oral pyogenic granuloma-A case series. Int J Cur Res Rev 4(24), 2012, 119-123.

[6]. Sangeetha Ramu, Chariotte Rodrigues. Reactive hyperplastic lesions of the gingiva: a retrospective study of 260 cases. World Jof Dentistry 3(2), 2012, 126-30.

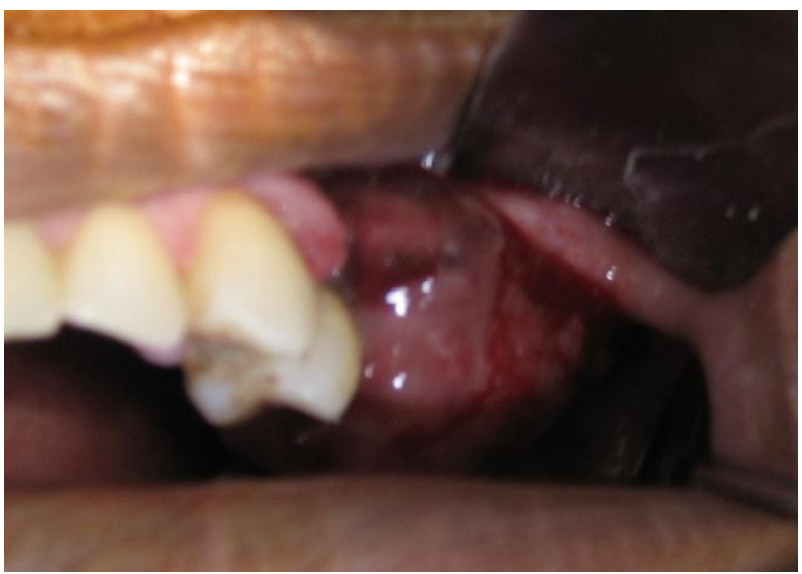

Figure 1: clinical appearance of the growth on maxillary left gingiva

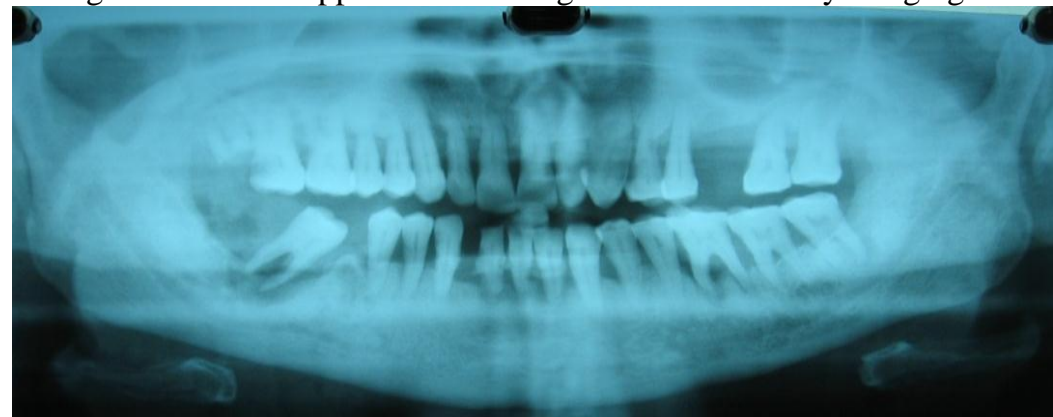

Figure 2: OPG showing generalized interdental bone loss 


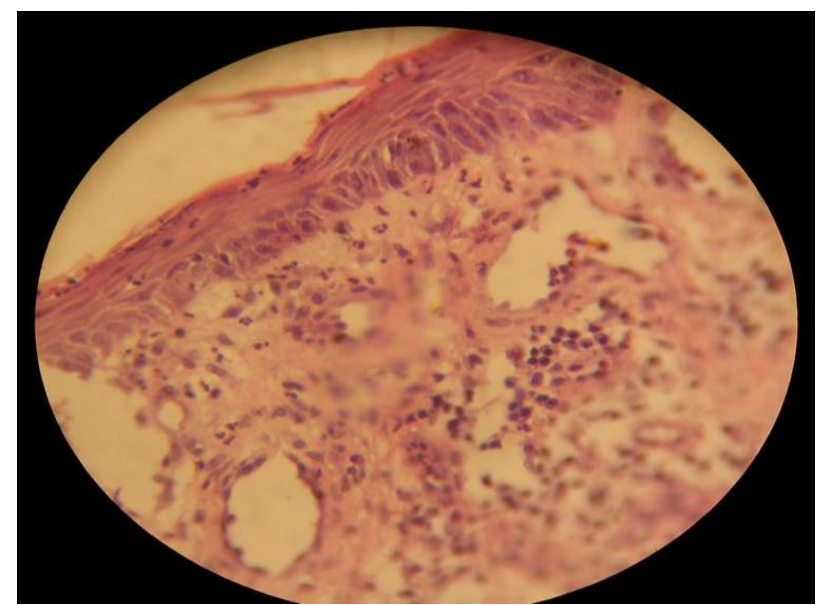

Figure 3: Photomicrograph of pyogenic granuloma

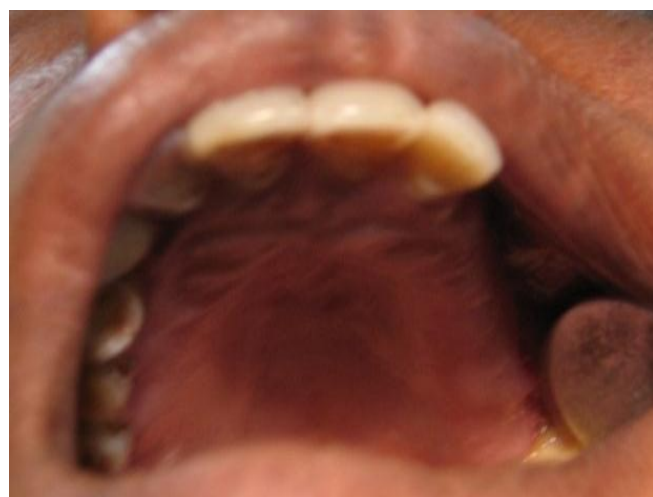

Figure 4: 9month post-op follow-up photograph 